\title{
Package Identifier with Attribute Identifier
}

National Cancer Institute

\section{Source}

National Cancer Institute. Package Identifier with Attribute Identifier. NCI Thesaurus.

Code C93889.

A unique symbol that establishes identity of the package. 